\title{
Haplotype mapping uncovers unexplored variation in wild and domesticated soybean at the major protein locus cqProt-003
}

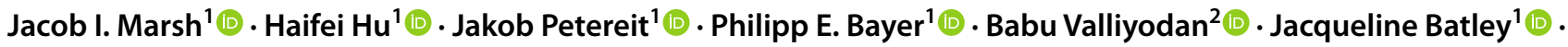 \\ Henry T. Nguyen ${ }^{3}$ (1) $\cdot$ David Edwards ${ }^{1}$ (1)
}

Received: 13 October 2021 / Accepted: 22 January 2022 / Published online: 9 February 2022

(c) The Author(s) 2022

\begin{abstract}
Key message The major soy protein QTL, cqProt-003, was analysed for haplotype diversity and global distribution, and results indicate $304 \mathrm{bp}$ deletion and variable tandem repeats in protein coding regions are likely causal candidates. Abstract Here, we present association and linkage analysis of 985 wild, landrace and cultivar soybean accessions in a pan genomic dataset to characterize the major high-protein/low-oil associated locus cqProt-003 located on chromosome 20. A significant trait-associated region within a $173 \mathrm{~kb}$ linkage block was identified, and variants in the region were characterized, identifying 34 high confidence SNPs, 4 insertions, 1 deletion and a larger 304 bp structural variant in the high-protein haplotype. Trinucleotide tandem repeats of variable length present in the second exon of gene Glyma.20G085100 are strongly correlated with the high-protein phenotype and likely represent causal variation. Structural variation has previously been found in the same gene, for which we report the global distribution of the $304 \mathrm{bp}$ deletion and have identified additional nested variation present in high-protein individuals. Mapping variation at the cqProt-003 locus across demographic groups suggests that the high-protein haplotype is common in wild accessions (94.7\%), rare in landraces (10.6\%) and near absent in cultivated breeding pools (4.1\%), suggesting its decrease in frequency primarily correlates with domestication and continued during subsequent improvement. However, the variation that has persisted in under-utilized wild and landrace populations holds high breeding potential for breeders willing to forego seed oil to maximize protein content. The results of this study include the identification of distinct haplotype structures within the high-protein population, and a broad characterization of the genomic context and linkage patterns of cqProt-003 across global populations, supporting future functional characterization and modification.
\end{abstract}

\section{Introduction}

Shifting climatic and ecological conditions threaten global food security at a time when the growing human population requires crop yields to increase an estimated $+50 \%$ to $+110 \%$ by 2050 (Alexandratos and Bruinsma 2012; Ray

Communicated by Volker Hahn.

David Edwards

dave.edwards@uwa.edu.au

1 School of Biological Sciences and Institute of Agriculture, University of Western Australia, Perth, WA 6009, Australia

2 Department of Agriculture and Environmental Sciences, Lincoln University, Jefferson City, MO 65101, USA

3 Division of Plant Sciences and National Center for Soybean Biotechnology, University of Missouri, Columbia, MO 65211, USA et al. 2013; Tilman et al. 2011; van Dijk et al. 2021). Domestication and improvement of major crops have led to genetic bottlenecks and reduced diversity due to strong selection for agronomic traits, especially in self-pollinating plant species such as soybean (Glycine max (L.) Merr.) (Hyten et al. 2006). Whilst intensive breeding efforts have increased crop productivity, it has left the regions of the soybean genome under selection with low genetic diversity in some modern breeding populations (Zhao et al. 2015). The lack of variation in these regions is particularly concerning, as they have proven to play important roles in plant function or morphology, and yet there is limited allelic variation remaining in modern lines for trait expansion and adaptation. When dissecting the genomic regions underlying agronomic traits it is important to look beyond traditional breeding populations and capture the full range of potential diversity. Fortunately in soybean, ancestral diversity persists in the wild progenitor Glycine soja (Siebold \& Zucc.) and exotic 
landrace populations that still harbour genomic variation at loci that are of value to breeders (Kofsky et al. 2018; Zhang et al. 2017).

Broad resequencing of soybean germplasm has provided a wealth of data for characterizing genetic diversity and for identifying genomic variation underlying agronomic traits (Fang et al. 2017; Torkamaneh et al. 2021a; Valliyodan et al. 2021; Zhou et al. 2015). In addition, the development of pangenomic datasets that increase variant and marker data accessibility across germplasm collections offers novel opportunities for plant breeding studies by providing the entire genetic content of soybean, including small and large structural variations, across global populations (Bayer et al. 2021; Liu et al. 2020; Torkamaneh et al. 2021b). Several studies have mapped important major effect genomic loci; for example disease resistance (Chang et al. 2016); SCN resistance (Patil et al. 2019); mycorrhizal colonization (Pawlowski et al. 2020); salt tolerance (Do et al. 2019); seed composition (Patil et al. 2018); and descriptive traits such as flower and pubescence colour (Bandillo et al. 2017). Genetic approaches incorporating whole-genome information with breeder friendly phenotyping (Reynolds et al. 2020) have allowed precise dissection of the genetic architecture of complex traits including seed composition in soybean. However, comprehensive bioinformatic analysis of key traits, such as seed compositional traits, is needed to capitalize on this fast accumulating wealth of soybean data for agronomic gain (Marsh et al. 2021).

Protein content in soybean is associated with a major quantitative trait locus (QTL); cqProt-003 on chromosome 20 that underwent a genetic bottleneck during domestication with selection for oil, at the cost of fixing a low-protein phenotype across modern breeding populations (Brzostowski et al. 2017). The region associated with cqProt-003 was first detected as a result of QTL mapping in an experimental population derived from crossing a domesticated G. $\max$ line with a wild $G$. soja individual (Diers et al. 1992). This seed protein QTL was later genetically mapped to a $3 \mathrm{cM}$ region on Linkage Group I (Nichols et al. 2006), before being located to an $8.4 \mathrm{Mb}$ genomic region on chromosome 20 (Bolon et al. 2010). Attempts over the following decade to refine the genomic locus responsible for seed composition variability have produced conflicting reports, in part due to the extensive linkage present across the region (Bandillo et al. 2015; Cao et al. 2017; Hwang et al. 2014; Vaughn et al. 2014). It has been suggested that a 321 bp structural variant (SV) in an exonic region of the gene Glyma.20G085100 (CCT motif family protein) may be responsible for the phenotypic effect (Fliege 2019). However, questions remain as to whether other variation is present in the high-protein haplotype, and the genomic context and the distribution of the high-protein haplotype across diverse international lines are largely uncharacterized.
Here we take a comprehensive approach to characterizing genomic variation at the cqProt003 locus using recently published high-quality pangenomic datasets from wild and domesticated soybean populations. This study aims to characterize the haplotype variation present within this region and evaluate the potential effects of allelic variants that may contribute to the high-protein phenotype. This will provide breeders and researchers with a detailed map of untapped variation in the cqProt-003 region that can serve as a roadmap for crop improvement.

\section{Materials and methods}

\section{Genotyping and additional genomic resources}

The SNP matrix was generated from 985 of the soybean lines in the previous soybean pangenome study (Bayer et al. 2021). Briefly, adapters of raw reads were removed using Trimmomatic v0.36 (Bolger et al. 2014). Clean reads were mapped to the Wm82.a4.v1 (Valliyodan et al. 2019) reference genome using BWA-MEM v0.7.17 with default settings (Li 2013) and duplicates removed by Picard tools (http:// broadinstitute.github.io/picard/). Reads were realigned by GATK (McKenna et al. 2010) v3.8-1-0 RealignerTargetCreator and IndelRealigner, followed by variant calling using GATK HaplotypeCaller. Then, indels and SNPs residing on unassembled scaffolds were split into separate files. The resulting SNPs were filtered $(\mathrm{QD}<2.0\|\mathrm{MQ}<40.0\|$ FS $>60.0 \|$ QUAL $<60.0 \|$ MQrankSum $<-12.5 \|$ ReadPosRankSum $<-8.0$ ) $t-$ remove low-quality SNPs. SNP loci with more than $10 \%$ missing data and less than $1 \%$ minor allele frequency were filtered out using VCFtools v3.0 (Danecek et al. 2011), resulting in 544,997 high-quality SNPs for chromosome 20. The filtered dataset included phasing and imputation of missing genotypes was conducted using BEAGLE v5.1 (Browning and Browning 2007). Variant annotation was predicted using SnpEff v3.0 (Cingolani et al. 2012). Nucleotide diversity $(\pi)$ and divergence (dXY) were calculated using pixy v1.0.4.beta1 using all invariant sites (Korunes and Samuk 2021), with all sites filtered to max-missing 0.9 and variant sites only filtered with - minQ 30 using VCFtools v3.0 (Danecek et al. 2011).

\section{Comparative genomics}

The Wm82.a4.v1 reference fasta sequence for the Glyma.20G085100 genic region $(31,724,592-31,729,626)$ was compared to the whole reference genomes of 13 legume species and 2 outgroup Rosids species: Glycine max (Valliyodan et al. 2019), Glycine soja \{Xie, 2019 \#370\}, Cajanus cajan \{Varshney, 2011 \#553\}, Phaseolus vulgaris (UI111 v1.1, DOE-JGI and USDA-NIFA, http://phytozome.jgi. 
doe.gov/), Phaseolus lunatus \{Garcia, 2021 \#554\}, Vigna unguiculata \{Lonardi, 2019 \#555\}, Vigna radiata \{Kang, 2014 \#556\}, Vigna angularis \{Yang, 2015 \#557\}, Lupinus albus \{Hufnagel, 2020 \#558\}, Lotus japonicus \{Sato, 2008 \#559\}, Cicer arietinum \{Varshney, 2013 \#560\}, Medicago truncatula \{Pecrix, $2018 \# 561\}$, Pisum sativa $\{$ Kreplak, 2019 \#562\}, Vitis vinifera (accessed in release 52 at http:// ftp.ensemblgenomes.org) \{Jailon, 2007 \#563\} and Arabidopsis thaliana $\{$ Lamesch, 2012 \#564\}. Nucleotide-Nucleotide BLAST 2.12.0 + ZZhang, $2000 \# 578\}$ was used with parameters -outfmt 6 -word_size 11. After running with the entire Glyma.20G085100 genic region as the query, the results were classified as part of the $304 \mathrm{bp} \mathrm{SV}$ region if Qrystart $>4000$ and Qryend > 4400. Results with an E-value less significant than E-10 and bitscore $<149$ were filtered out.

\section{Insertions, deletions and structural variation}

Insertions and deletions were recoded to biallelic sites to enable straightforward linkage calculation with SNPs. Insertions were split from deletions based on the relative lengths of the alternate alleles compared to the reference using BCFtools norm - $\mathrm{m}$ ( $\mathrm{Li}$ et al. 2009). The insertion and deletion files were then filtered (-minQ 30 -max-missing 0.9 -non-ref-af-any 0.01) with VCFtools v3.0 (Danecek et al. 2011). Multiallelic records were then re-merged for insertions and deletions before being recoded to biallelic sites with all variant alleles of the same type (i.e. insertion or deletion) represented by a single proxy alternate polymorphism (e.g. Adenine), whilst the reference allele was also recoded to a different proxy allele (e.g. Tyrosine). For chromosome 20, 35,826 Insertions and 55,512 deletions were kept following filtering and recoding. Recoded biallelic insertions and deletions were then merged with filtered SNPs in separate files in order to preserve 4540 multiallelic sites with both insertions and deletions. Whilst recoded InDels were used to determine linkage between variants, downstream analysis, such as for the trinucleotide insertion of interest, was done using the original filtered, unimputed information. The presence and absence for the 304 bp SV were classified from significant drops (8-50X to $0-1 \mathrm{X})$ in per-base coverage in the BAM file across the Glyma.20G085100 genic region using mosdepth v0.2.6 (-b 1 -Q 10) (Pedersen and Quinlan 2018).

\section{Phenotype collection and metadata}

The protein and oil phenotypic data used in this study were obtained from the USDA-Soybean Germplasm Collection general evaluation trials which includes morphological, agronomic and seed composition data sets. These field evaluations were conducted at different locations based on the maturity group where some of them were grown for several years and the total protein and oil concentration were measured using various methods. The protein and oil concentration measurements of the soybean accessions with yellow seed coat were conducted using the near-infrared reflectance method on whole seed sample. The dark or pigmented seedcoated soybean samples were analysed for total protein content using the Kjeldahl method and the seed oil content using the Butt method (Bandillo et al. 2015). There are several reports using these data sets for genotype-phenotype association studies and identification of oil and protein content genes or QTL in soybean (Bandillo et al. 2015; Hwang et al. 2014; Jarquin et al. 2016; Vaughn et al. 2014). List of accessions used in this study with the available protein and oil data is available in Table S6.

\section{Genome-wide association analysis}

Association analysis was performed using the FarmCPU (Liu et al. 2016) and MLM (Price et al. 2006) methods implemented in rMVP (Yin et al. 2021). The GWAS included data from all 985 lines for protein, and the subset with data available of 945 for oil (Table S6) with imputed and unimputed data (Table S1, Table S3). Population structure was controlled using the first three PCAs based on the marker data. The significance threshold was determined by $0.05 /$ marker size.

\section{Linkage disequilibrium analysis}

The linkage disequilibrium heat map was generated using LDBlockShow (Dong et al. 2020) for $\mathrm{R}^{2}$ and $\mathrm{D}^{\prime}$ with block type set to the Gabriel method for the D' figure (Gabriel et al. 2002), the input for the Manhattan plot was the protein GWAS FarmCPU results from the filtered, unimputed SNP data. The approach used for the detailed haplotype block information was the confidence interval definition (Gabriel et al. 2002) implemented in PLINK1.9 (Purcell et al. 2007) with default parameters. Pairwise $\mathrm{R}^{2}, \mathrm{D}^{\prime}$ and allele frequency values were obtained for sites using PLINK1.9 (Purcell et al. 2007) by first using -show-tags with -tag-r2 0.9 to identify sites in linkage with the GWAS-SNP, before generating detailed information with this list of sites with $-\mathrm{r} 2$ dprime. Site missingness information was generated using VCFtools v3.0 (Danecek et al. 2011).

To avoid false positive correlations, linkage analysis was conducted on filtered, unimputed SNPs with recoded biallelic insertions and deletions. Linkage requirements to include insertions/deletions in the cqProt-003 haplotype were relaxed to $\mathrm{R}^{2}>0.85$ to compensate for the loss of information from recoding with merged variants. For 
the $304 \mathrm{bp} \mathrm{SV}$, individuals with presence/absence were recoded the same way for linkage testing, and the relatively higher impact larger variations can have compared to point mutations. Linkage between SNPs and insertions/ deletions was testing between the presence of an insertion/ deletion of any length compared to the reference allele, rather than specific variants for multiallelic sites.

\section{Site-centric haplotyping}

Site-centric haplotyping was conducted using HaplotypeMiner software (Tardivel et al. 2019). The genomic input was generated using the filtered, imputed SNP dataset converted to hapmap format using Tassel 5 (Bradbury et al. 2007), with heterozygotes set to missing, from which only sites within the $173 \mathrm{~kb}(31,604,127$ to $31,777,346)$ region were retained. The R2 measure was ' $\mathrm{r} 2 \mathrm{vs}$ ' with a kinship file generated using the Endelman \& Jannink (Endelman and Jannink 2012) method implemented in Tassel 5 (Bradbury et al. 2007) and a structure file generated using fastStructure (Raj et al. 2014) with optimal $\mathrm{K}=4,-$ prior $=$ simple. The cluster $\mathrm{R} 2$ was set to ' $\mathrm{r} 2$ ' only to reduce the computational load. The 'gene' region provided was the start and end position of the haplotype block defined by PLINK that contains the GWAS-SNP which represents general linkage around this specific site; start: $31,621,924$ end: $31,644,477$. The following parameters were applied: max_missing_threshold $=0.6$, max_het_threshold $=0.3$, min_alt_threshold $=0.01, \min _{-}$ allele_count $=4$, marker_independence_threshold $=0.9$, with a range of cluster thresholds tested using the clustree R package (Zappia and Oshlack 2018). Segmentation between cluster groups was assessed by performing PCA implemented in the factoextra $\mathrm{R}$ package which provided input for UMAP (McInnes et al. 2018) with the following parameters: -pc 7 -nn 15 -md 0.001.

Linkage of other sites with the representative marker SNPs that define the haplotypes was conducted independently using PLINK1.9 (Purcell et al. 2007) with -tag-r2 0.9 on the unimputed dataset. Welch's independent twosample $t$ test assuming unequal variance (Welch 1947) was used to test for significant differences of protein content between individuals across haplotype groups and marker groups. Six marker groups that were co-inherited in every haplotype population with another representative marker were removed to reduce redundancy, keeping only one representative marker; a further six marker groups were removed which either included only five or fewer SNPs or differed from another marker group by only one SNP. Graphical summaries of the haplotype populations and marker groups with phenotype information were conducted using ggplot2 in R v4.04 (R Core Team 2021).

\section{Results}

\section{Phenotype association analysis}

A genome-wide association study (GWAS) for protein content was conducted on chromosome 20 using 985 accessions, including 131 wild lines, 708 landraces, 44 old cultivars and 102 modern cultivars, for which phenotypic data was available from the UWA SoyPan dataset (soybase.org). GWAS was tested using different combinations of unfiltered, filtered, unimputed and imputed data (Figure S1, Figure S2, Figure S3, Table S1). The results using the FarmCPU method and the unimputed dataset indicate a highly significant site associated with protein content located at position 31,649,589 in the Wm82.a4.v1 reference genome (Valliyodan et al. 2019) (Table S1); however, a site $17 \mathrm{~kb}$ upstream $(31,632,556)$ was detected when using imputed data (Figure S4, Table S1). Given the $9.3 \%$ missing variant information from lines that did not align at position $31,649,589$ (Figure S5, Table S2), the SNP at 31,632,556 (hereafter referred to as "GWASSNP") was taken as the most confident GWAS result using this method. Using the MLM method, no sites registered above the significance threshold $\left(p<1.0 \mathrm{E}^{-08}\right)$; the most significant site was at $31,604,127$ bp with a p-value of $3.15 \mathrm{E}-07$, with 13 of the top 20 sites all within the $104 \mathrm{~kb}$ range of $31,604,126-31,708,981 \mathrm{bp}$ (Table S1). GWAS for oil content was conducted on the same dataset using the FarmCPU method and 945 individuals (Table S3). A significant site corresponding to decreased seed oil ( $p=3.4 \mathrm{e}-09$ ) was detected at $31,687,912 \mathrm{bp}$, at which the 183 individuals with the alternate allele displayed a mean oil content $32.7 \%$ lower ( $6.1 \mathrm{~g}$ oil per $100 \mathrm{~g}$ seed weight) than the 743 individuals conforming to the reference.

\section{Delimiting the cqProt-003 region}

The chromosomal region associated with the protein phenotype was defined from linkage disequilibrium surrounding the GWAS-SNP. Significant, though non-continuous linkage, defined using $\mathrm{R}^{2}$, is apparent in large blocks across a wide $300 \mathrm{~kb}$ region (Fig. 1), with a block of LD surrounding the GWAS-SNP from approximately $31,600,000-31,800,000$. Linkage blocks in the region defined using the confidence interval approach are relatively continuous from 31,601,763-31,732,110 bp, becoming more disconnected in adjacent regions (Figure S6, Table S4).

Linkage with the GWAS-SNP assessed using $\mathrm{R}^{2}$ remained relatively high at long distances, with a value above 0.7 beyond $1 \mathrm{Mbp}$ downstream (Figure S7), though 
Fig. 1 Linkage disequilibrium (R2) between SNPs across $450 \mathrm{~Kb}$ region surrounding the significant protein GWAS hit at $31,632,556$. The black triangle represents the $173 \mathrm{~kb}$ cqProt-003 region defined from decay of linkage with the GWAS-SNP only

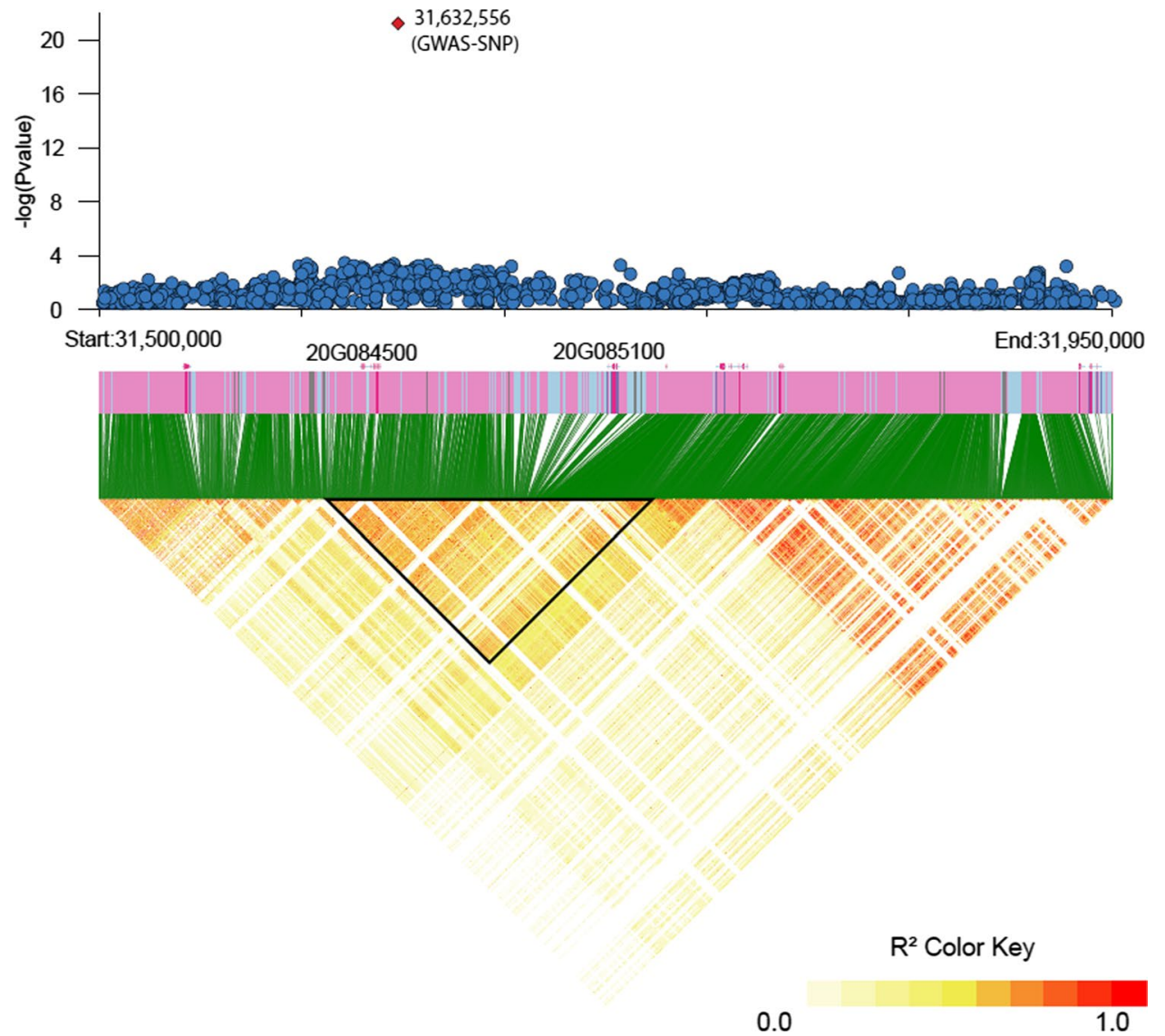

potentially indicating structural diversity, problematic read alignments or mis-assembly in the Wm82.a4 reference.

\section{Patterns of linkage present within the region}

Haplotyping the broader genomic context of cqProt-003 was conducted using SNPs in the $173 \mathrm{~kb}$ region to identify and characterize all distinct linkage patterns, beyond simply those associated with protein content. Haplotyping the cqProt-003 region produced distinct groups of internally linked markers as well as groups of individuals with different haplotype combinations of these marker groups. The haplotyping resulted in nine groups of individuals (A-G combinations) that contained different combinations of six groups of markers (Fig. 2), which include 370 SNPs that are in tight linkage with one of the different representative sites (M01-M06) (Fig. 3, Figure S10, Figure S11, Figure S12, Figure S13, Table S5, Table S6). Comparing the phenotype score between individuals from different haplotype groups indicates a significant elevation of protein content $\left(p=7.47 \mathrm{e}^{-14}\right)$, with a mean increase of $3.32 \mathrm{~g} / 100 \mathrm{~g}$ seed weight for landrace individuals with the alternate allele for marker group M01; conversely, oil content displays a mean decrease of $2.66 \mathrm{~g} / 100 \mathrm{~g}$ seed weight (Tables S7, Table S8). These high-protein/low-oil haplotypes (B-G) are comprised 


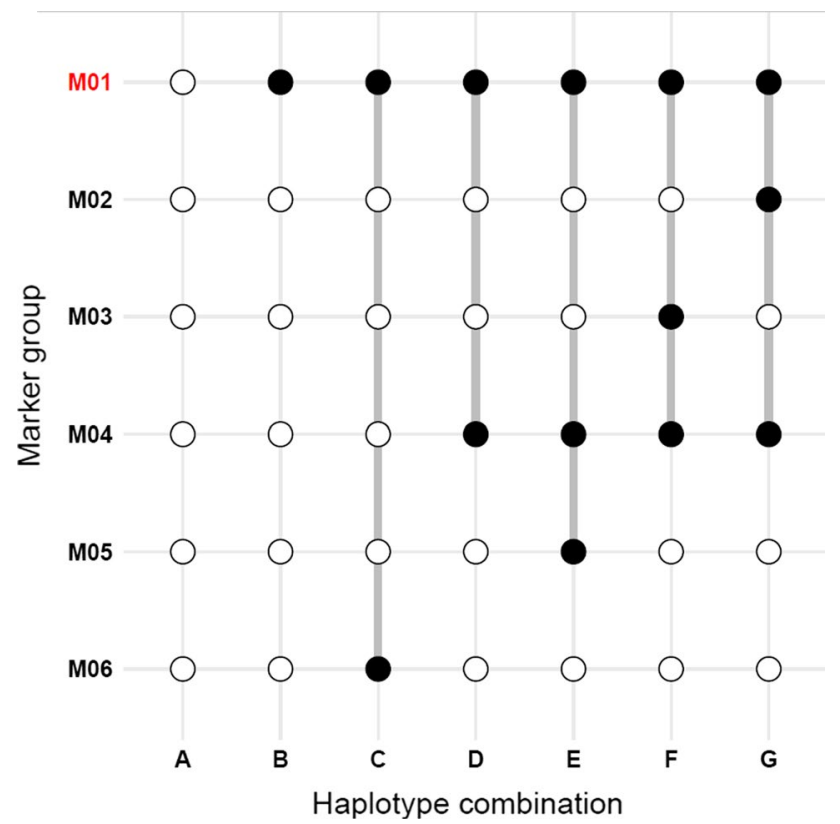

Fig. 2 Region-centric haplotypes for $173 \mathrm{~kb}$ region indicating alternate alleles (black dots) for clustered markers (M01-M06) that define unique haplotype combinations (A-G) in the $173 \mathrm{~kb}$ region of interest; white dots indicate reference alleles present. The protein-associated haplotype, M01, is highlighted in red (colour figure online)

mostly of wild and landrace individuals, compared to haplotype group A which is predominantly domesticated (Fig. 4). Wild individuals display consistently lower oil content with highly variable protein content; however, landrace individuals display a high degree of oil variability within and between haplotypes (Fig. 4). Notably, haplotype group $\mathrm{C}$ represents an exclusively landrace population $(\mathrm{n}=21$, $\mu$ Protein $\left.=48.762, S^{2}=6.902\right)$ that remains consistent with the high-protein phenotype seen in other haplotype populations that contain a mixture of wild and landrace individuals (Fig. 4, Table S7).

Genomic variation across the region is primarily present in individuals with the alternate allele in the M01 marker group (Figs. 2, 3). In the $173 \mathrm{~kb}$ genomic region, nucleotide diversity $(\pi)$ is $3.85 \mathrm{E}^{-3}$ among individuals with the alternate allele for the M01 marker group, compared to $\pi=3.24 \mathrm{E}^{-4}$ with the reference allele. This represents a significant drop of diversity in the $173 \mathrm{~kb}$ region for the population with the M01 reference allele that possess an average diversity $(\pi)$ of $1.74 \mathrm{E}^{-3}$ across the entire genome. In contrast, the population of individuals with the alternate allele at M01 has only slightly elevated genetic diversity in the region relative to the mean across the whole genome $\left(\pi=3.60 \mathrm{E}^{3}\right)$. Increased genetic diversity in the region appears to be contained in the predominantly wild populations; of the alternate M01 haplotypes, only the landrace group $\mathrm{C}$ possesses lower levels of diversity consistent with the primarily domesticated haplotypes A and B (Table S7). Furthermore, divergence in the $173 \mathrm{~kb}$ region between groups A-C is far lower than groups D-G with the alternate M04 marker group (Figure S14, Figure S15), which includes 157 SNPs, though has a slightly lower association with seed protein than M01 (Fig. 3).

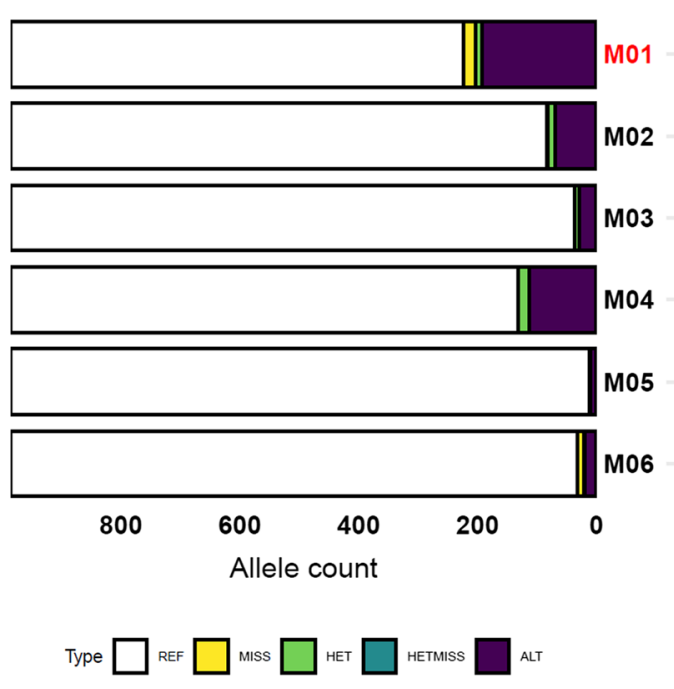

Fig. 3 Allele frequencies for the representative markers (left) and summary of supporting markers in each group (right). Marked in red is the marker group containing candidate variants for the high-protein haplotype (M01). Each dot represents a SNP within a given marker group, for which is displayed the average protein difference between individuals who have the alternate vs reference allele, coloured whitepurple by that site's alternate allele frequency. All alternate alleles

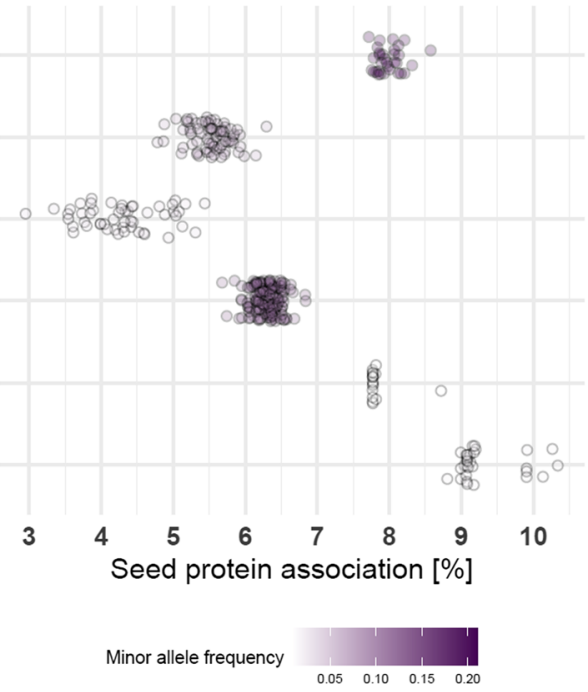

depicted are positively associated with seed protein, compared to the reference allele. REF refers to the frequency of homozygous reference alleles at the marker groups' representative site, similarly for homozygous missing (MISS), heterozygous (HET), sites with one missing allele (HETMISS) and homozygous alternate alleles (ALT) (colour figure online) 


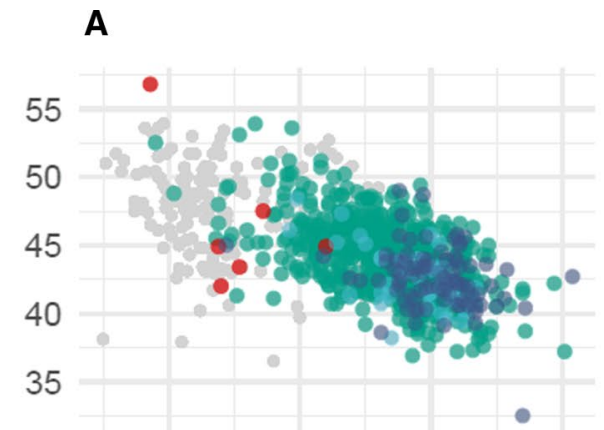

B

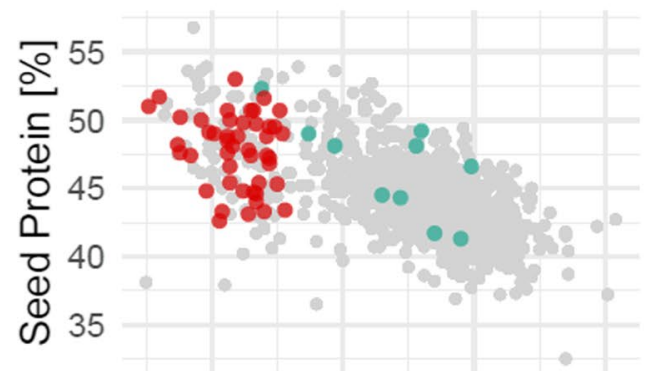

E

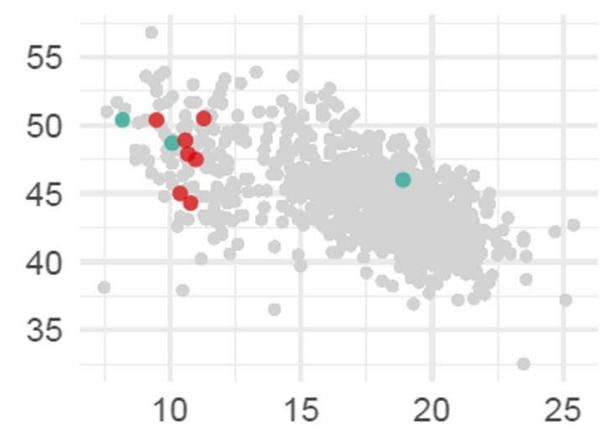

\begin{tabular}{cccccc}
\hline Hap & Total & Wild & Landrace & Old Ctvr & Mod Ctvr \\
\hline A & 780 & 7 & 620 & 43 & 85 \\
B & 58 & 47 & 10 & 0 & 0 \\
C & 21 & 0 & 19 & 0 & 0 \\
D & 5 & 3 & 2 & 0 & 0 \\
E & 10 & 7 & 3 & 0 & 0 \\
F & 34 & 26 & 2 & 1 & 1 \\
G & 77 & 41 & 35 & 0 & 1 \\
\hline
\end{tabular}

C

D

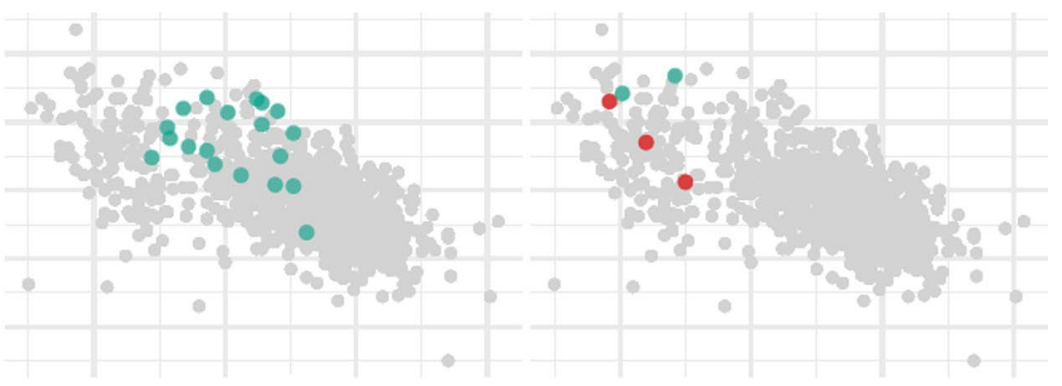

$\mathbf{F}$

G

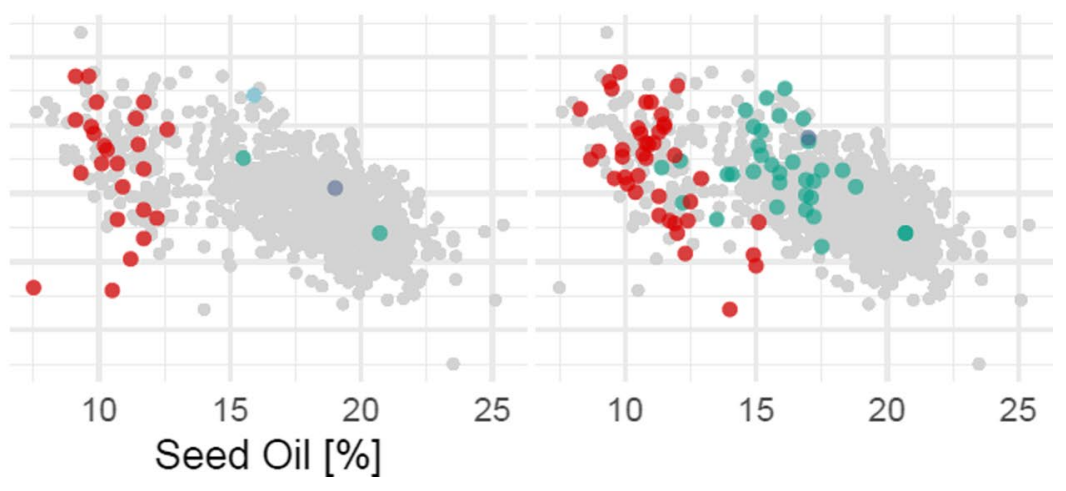

Fig. 4 Phenotype associations of haplotype combinations (A-I) with population breakdown. Each dot represents an individual possessing a given haplotype population (A-G), coloured by level of domesti-

\section{Trinucleotide repeat expansion found in the high-protein haplotype}

The haplotype of 40 variants in linkage with the GWASSNP (Table S9) was further characterized. Four insertions and a single deletion were also found to be in tight linkage $\left(\mathrm{R}^{2}>0.85\right)$ with the GWAS-SNP, warranting their inclusion in the high-protein haplotype (Table S9). Of the 39 small- and medium-sized variants classified as in the cqProt-003 haplotype, 33 were in intergenic regions and five were adjacent to genes (Table S9). There were no variants detected from the haplotype in coding regions for two of the three genes in the $173 \mathrm{~kb}$ region: Glyma.20G084500 and Glyma.20G085250. However, for the final gene, Glyma.20G085100.1, a multiallelic insertion cation: wild, landrace, old cultivar ('Old Ctvr') and modern cultivar ('Mod Ctvr'); grey points include all individuals for reference

at 31,727,019 bp is located in the second exon (Table S10). The mutations at this locus appear to be predominantly trinucleotide tandem repeat expansions of 'CAA', which are conservative in-frame insertions of Asparagine; however, there are four individuals who reported either disruptive inframe insertions or frameshift variants (Table 1). Immediately downstream of this site are five invariant 'CAA' copies from $31,727,020-31,727,035$ common to all soy individuals, which the insertions expand. The phenotype scores for individuals that contain homozygous tri-allelic insertions are significantly elevated, with an average protein score increase of $7.9 \%$ compared to individuals without an insertion (Table 1), consistent with the cqProt-003 haplotype. The mean protein scores for individuals with each alternate sequence at the tandem repeat locus were all higher than the 
Table 1 Summary of individuals with different alleles present at the trinucleotide repeat locus starting at 31,727,019, including 'CAA' copy number, annotation, individual count and mean protein content

\begin{tabular}{llll}
\hline Trinucleotide copies & Annotation & Individuals & Mean protein \\
\hline 5 & (reference) & 787 & 43.86 \\
6 & CIF & 2 & 49.85 \\
7 & CIF & 10 & 46.67 \\
8 & CIF & 8 & 45.64 \\
9 & CIF & 21 & 46.75 \\
10 & CIF & 13 & 48.19 \\
11 & CIF & 24 & 47.19 \\
12 & CIF & 46 & 46.68 \\
13 & CIF & 30 & 48.97 \\
14 & CIF & 1 & 50.20 \\
Other & 2xDIF; 2×FSV & 4 & 44.78 \\
Heterozygous repeats & N/A & 11 & 47.09 \\
Single repeat & N/A & 15 & 47.03 \\
Missing & N/A & 13 & 46.92 \\
Total & N/A & 985 & 44.54 \\
\hline
\end{tabular}

The 'other' row refers to four individuals with insertions at this locus that did not fit the typical tandem repeat structure; 'heterozygous repeats' row refers to individuals that are heterozygous at the locus with two different insertions; 'single repeat' refers to individuals that are heterozygous with one copy of any insertion and one reference allele; 'other' refers to individuals that are homozygous for an insertion that is not a trinucleotide repeat of 'CAA'; 'missing' refers to individuals with one or both missing alleles. Annotations include conservative in-frame insertion (CIF), disruptive in-frame insertion (DIF) and frameshift variant (FSV)

mean for individuals without an insertion at this site; however, sample sizes are insufficient for confident association testing between these alleles (Table 1). In landrace individuals, $82.3 \%$ with homozygous alternate tandem repeat possessed seven or eight additional repeats, compared to $25.8 \%$ in wild individuals, which have more diversity (Fig. 5).

\section{Trait associated structural variation}

Further structural variation was confirmed in the same gene in the $173 \mathrm{~kb}$ region, Glyma.20G085100 gene, with a $304 \mathrm{bp}$ deletion (relative to $\mathrm{Wm} 82$ ) (Figure S16). The deletion spans from $31,728,619$ to $31,728,923 \mathrm{bp}$, truncating $94.8 \%$ of the fourth exon, and $56.4 \%$ of the first of two $3^{\prime}$ UTRs (Table S10). This deletion is present in 194 lines that have a mean protein content $7.9 \%$ greater than those with the reference sequence (Table S6), consistent with the highprotein haplotype. There is significant overlap between the trinucleotide insertions and the 304 bp deletion: 152 of the 194 individuals with the 304 bp deletion possess a homozygous trinucleotide insertion, and all but one individual with a homozygous trinucleotide insertion had the 304 bp deletion (Table S6). The 304 bp deletion is linked to the GWASSNP with $\mathrm{R}^{2}=0.918$ and $\mathrm{D}^{\prime}=0.958$, and it is most tightly linked with the SNP at position $31,680,574 \mathrm{bp}\left(\mathrm{R}^{2}=0.952\right.$, $\left.\mathrm{D}^{\prime}=0.984\right)$. The start position for the $304 \mathrm{bp}$ deletion, 31,728,619 in Wm82.a4.v1 (Valliyodan et al. 2019), coincides with a smaller insertion in some lines (Table S6). The most common insertion at this site is $25 \mathrm{bp}$, which appears exclusively in 30 of the 194 individuals with the 304 bp deletion $(\mu$ Protein $=48.29$; Table S6).

No significant (MAF $>0.05)$ InDel sites were identified in the syntenic cqProt-003 region from a Korean collection of 855 soybean individuals (Kim et al. 2021). However, the InDel data available from a recent soybean pangenome (Liu et al. 2020) suggest additional variation may be present in the Glyma.20G085100 genic region (annotated as ZH13_20G075300; Table S11). This dataset similarly contains trinucleotides with 3-8 'CAA' inserted repeats across $5.3 \%$ of individuals; however, they do not identify the remaining six other rare alternate alleles (Table 1, S11). Beyond the trinucleotide tandem repeat insertions, Liu et al.'s data presents similar structural variation in the genic
Fig. 5 Frequency of trinucleotide insertion alleles at the $31,727,019$ locus, by domestication status. The 'het' column refers to individuals that are heterozygous at the locus with two different repeat insertions; 'het1' column refers to individuals that are heterozygous with a single copy of any repeat insertion and one reference allele; other refers to individuals that are homozygous for an insertion that is not a typical trinucleotide repeat of 'CAA'

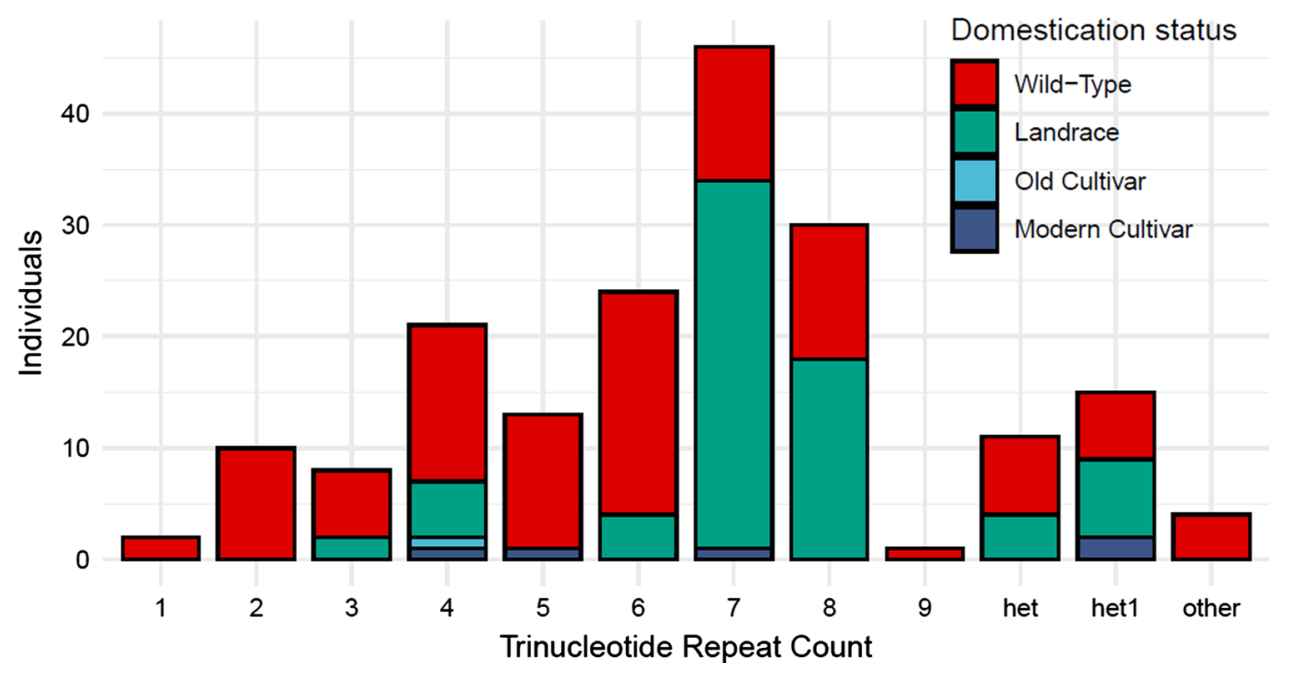


region of Glyma.20G085100 (Table S11). The W05, W01, W02, W03 and SoyC12 accessions contain a 318 bp (302 bp for W03) deletion relative to ZH13 and WM82 cultivars, significantly truncating the upstream portion of the fourth exon for ZH13_20G075300/Glyma.20G085100 (Table S10-11). Individuals with 318 bp deletions in the Liu et al. dataset are present in our data with 304 bp deletions; however, no deletion in the Glyma.20G085100 gene was identified for W05 using the same genomic data from Liu et al. aligned to the Wm82.v1.a4 reference.

\section{Homology with other species and regions}

Local alignment searches for the nucleotide sequence of the gene containing both the trinucleotide insertions and SV, Glyma.20G085100, revealed homologous sequences closely resembling the $304 \mathrm{bp}$ deleted sequence are highly prevalent throughout the domesticated and wild soybean genomes (Table S12). In addition, more distant homologs for the 304 bp sequence were identified in abundance across the common pea (Pisum sativum) and barrel medic (Medicago truncatula) genomes, and less frequently in the chickpea (Cicer arietinum) genome (Table S12). Excluding the $304 \mathrm{bp}$ region, the rest of the Glyma.20G085100 gene possessed fragments with homologous sequences in all of the 13 legumes aligned against, though no homologous sequences in Arabidopsis thaliana and grape (Vitis vinifera) (Table S13). The previously reported paralog for Glyma.20G085100 gene, Glyma.10G13440 \{Brown, 2021 \#549\}, does not contain a homologous sequence for the 304 bp SV (Table S13); whilst the second exon of Glyma.10G13440 does contain six CAA repeats, starting at position 36,191,376 in Wm82. a4.v1, we could not identify InDel variation across the population at this locus indicative of expansion.

\section{Discussion}

Whole-genome resequencing datasets from global domesticated and wild soybean populations have provided an opportunity for the detailed characterization of the cqProt-003 protein-associated region, to identify patterns of variation and identify candidate causative variants. We have refined the genomic interval underlying significant protein and oil variability to a $173 \mathrm{~kb}$ region. The region defined in our study sits in the centre of a broader $550 \mathrm{~kb}$ 'fourth block' previously identified using USDA $50 \mathrm{k}$-SNP chip genotyping (Bandillo et al. 2015); however, the peak SNP in that block is approximately $400 \mathrm{~kb}$ downstream from the GWAS-SNP identified in our study. A GWAS conducted on 279 Chinese soybean cultivars defined a $960 \mathrm{~Kb}$ block on chromosome 20 associated with oil (Cao et al. 2017). This seed oil QTL region starts $7 \mathrm{~kb}$ upstream of the end of the $173 \mathrm{~kb}$ region associated with protein we report (Cao et al. 2017). More recently, an $839 \mathrm{~kb}$ linkage block identified in a GWAS of seed protein, oil and amino acids using USDA accessions from maturity groups I to IV is more distant, located $1.5 \mathrm{Mb}$ downstream from our region (Lee et al. 2019). The strong relationship between oil and protein in this region is apparent, indicating that the primary utility of the cqProt-003 locus is for breeders who are willing to forego oil content for higher seed protein, reflecting increasing demand for plant protein for human consumption. The inverse effect could be explained in part by the competing metabolic demands of protein and oil synthesis (Popovic et al. 2012); however, further dissection of how the cqProt-003 locus directly impacts absolute levels of yield components is required.

Our results benefit from higher SNP density, InDel data, and genome sequence data sufficient for the accurate detection of a broad range of SVs. This has allowed us to move beyond marker/trait association to detailing shared haplotypes with specific variants linked to trait variation at the cqProt-003 locus. The characterization of haplotype structures in the $173 \mathrm{~kb}$ context of cqProt- 003 provides a detailed overview of the local landscape of linkage and genomic variation. Our results show that genomic diversity has been significantly reduced by selection for the high oil/low-protein haplotype and largely lost in modern breeding pools. Genomic variants are in high linkage throughout the region, which limits the production of novel combinations, and most of the linked clusters of variants (M02-M06) appear to display variation only when the alternate alleles are present in the candidate marker group that is most strongly associated with protein content (M01). The lack of variation in individuals containing the Williams 82-like alleles for the M01 group appears to be the result of a strong domestication bottleneck for the high oil/low-protein phenotype in domesticated lines. Further functional characterization of the variation across the different haplotype populations (B-G) is needed to explore how different allelic combinations modulate the effect of cqProt-003 on seed compositional traits.

We identified two candidate variants located in Glyma.20G085100, providing additional evidence that this gene is likely involved in the high-protein phenotype. We located a $304 \mathrm{bp} \mathrm{SV}$ in the fourth exon that is associated with the low-protein phenotype. This represents an insertion in modern lines relative to wild progenitor populations, and this insertion is likely to modify the expression and/or function of the Glyma.20G085100 gene. Previous research that first identified this SV focused in the structural variation between two lines, PI468916 (HNY.50) and Williams 82 , before screening a population of 302 individuals using PCR with markers targeting a CCT domain (Fliege 2019). They conclude that this SV is the result of the insertion of a transposon fragment in low-protein domesticated populations, rather than a deletion in high-protein lines. Whilst 
further validation is needed, they provide preliminary results that the insertion could have a gain of function effect which can be counteracted by dampening the expression of Glyma.20G085100 using transgenics to increase protein in domesticated lines. However, there has been limited exploration regarding its genomic context and its distribution across different soybean populations. Discrepancies regarding the size of the SV (321 bp vs $304 \mathrm{bp)}$ ) are likely due to different assemblies, Wm82 compared to ZH13 (Liu et al. 2020), and because the PCR fragment amplified by Fliege et al. included $634 \mathrm{bp}$ of the genomic context which likely included other variation. The abundance of sequences homologous to the $304 \mathrm{bp} \mathrm{SV}$ in the inverted repeat-lacking clade of legumes (IRLC) indicate conserved function. However, complete absence of homologous sequences to the $304 \mathrm{bp}$ in more closely related clades such as Phaseoleae is evidence it has been selected against in many species; and its absence in more distantly related Leguminosae and Rosids species may suggest it is specific to Papilionoideae. We uncovered additional variation in coding regions, including a $25 \mathrm{bp}$ insertion at the start of the $304 \mathrm{bp} \mathrm{SV}$ that suggests multiple distinct variant combinations in Glyma.20G085100 could have unique influences on seed composition. We identified a trinucleotide repeat expansion of up to nine additional Asparagine copies in the second exon of Glyma.20G085100 that is tightly linked with the high-protein phenotype. The low-protein allele possesses only five tandem repeat copies and is seen in nearly all domesticated lines though is rare in wild progenitor populations. Trinucleotide repeat expansions are highly mutagenic structures that have been associated with degenerative diseases in humans (Nageshwaran and Festenstein 2015), especially when present in coding regions, where they can have significant impacts on protein structure (Figura et al. 2015), such as in the case of Huntington's disease (Shacham et al. 2019). Trinucleotide repeat expansions have also been implicated in temperaturesensitivity adaptation in Arabidopsis thaliana (Tabib et al. 2016), though remain largely unstudied in the plant kingdom (Zhu et al. 2021). The paralog of Glyma.20G085100, Glyma.10G13440, contains a similar number of trinucleotide copies (six) as is widely found in low-protein individuals (five). The lack of copy number variation at the repeat locus in Glyma.10G13440 suggests this may represent a threshold for mutability, providing additional evidence that the capacity for expansion has been lost for Glyma.20G085100 in most domesticated soy populations. Our finding may represent the first case of causal short tandem repeat variation in a coding region underlying phenotypic effect in soybean. We hypothesize that prior to domestication there may have been balancing selection to maintain a higher number of trinucleotide repeats that may be needed for a function that results in a high level of protein or oil. The repressed repeat count seen across most domesticated populations may be a significant loss for the adaptive potential of this gene that likely underpins a large degree of trait variability.

\section{Conclusion}

We have refined the linked region associated with the highprotein phenotype, defined haplotype structures within this region, thoroughly examined the high-protein haplotype, assessed the untapped variability within the population with this haplotype and identified likely causal genomic candidates. The variants in the high-protein haplotype act as high confidence markers for the high-protein haplotype, which can support low-cost genomic inference of the cqProt-003 trait. The causal candidates, trinucleotide insertions and structural variation in the Glyma.20G085100 gene, demand validation for impacts on plant phenotype. Furthermore, a key gap in our understanding still remains regarding the role in seed morphology and development of the additional proteins produced by individuals with the high-protein haplotype. A deeper understanding of the functional pathways involving the Glyma.20G085100 gene could open the door to further optimization of the cqProt-003 locus for agronomic gain using gene editing technology such as CRISPR. These findings draw attention to the lack of diversity in modern breeding lines at this important locus for seed composition, and the potential to exploit the natural variability remaining in exotic landraces and wild populations (haplotype populations B-G) to provide breeders with additional tools for producing protein-rich soy in a world with increasing nutritional demands.

Supplementary Information The online version contains supplementary material available at https://doi.org/10.1007/s00122-022-04045-8.

Acknowledgements The authors thank Australian Research Council for funding support through projects DP200100762, DP210100296, LP160100030 and DE210100398. This work was supported by resources provided by the Pawsey Supercomputing Centre with funding from the Australian Government and the Government of Western Australia. JIM would like to thank Marc-André Lemay for his suggestions regarding parameter optimization with the HaplotypeMiner program.

Author's contribution DE supervised the overall project; JM conceived the research and wrote the manuscript with input from $\mathrm{HH}, \mathrm{PEB}, \mathrm{BV}$ and HTN; JIM and HH conducted the bioinformatics analysis, with contributions from JP; JP, PEB, JB and DE contributed to the editing of the manuscript.

Funding Open Access funding enabled and organized by CAUL and its Member Institutions.

Data availability All code generated for this study is available at DOI: github.com/JacobIMarsh/cqProt-003. Supplementary Tables and Figures are available alongside all data generated for this study at http:// www.appliedbioinformatics.com.au/index.php/Soybean with VCF 
files for raw, filtered, imputed SNP and filtered InDels recoded as biallelic sites, and files used as input for HaplotypeMiner. JBrowse (Buels et al. 2016) genome visualization of the 40 variants identified in the cqProt-003 high-protein haplotype can also be accessed at http://appli edbioinformatics.com.au/soybean_protein/.

\section{Declarations}

Conflict of interest The authors declare no conflict of interests.

Open Access This article is licensed under a Creative Commons Attribution 4.0 International License, which permits use, sharing, adaptation, distribution and reproduction in any medium or format, as long as you give appropriate credit to the original author(s) and the source, provide a link to the Creative Commons licence, and indicate if changes were made. The images or other third party material in this article are included in the article's Creative Commons licence, unless indicated otherwise in a credit line to the material. If material is not included in the article's Creative Commons licence and your intended use is not permitted by statutory regulation or exceeds the permitted use, you will need to obtain permission directly from the copyright holder. To view a copy of this licence, visit http://creativecommons.org/licenses/by/4.0/.

\section{References}

Alexandratos N, Bruinsma J (2012) World agriculture towards 2030/2050: the 2012 revision. In: ESA (ed). FAO, Rome.

Bandillo N, Jarquin D, Song Q, Nelson R, Cregan P, Specht J, Lorenz A (2015) A population structure and genome-wide association analysis on the USDA soybean germplasm collection. Plant Genome. https://doi.org/10.3835/plantgenome2015.04.0024

Bandillo NB, Anderson JE, Kantar MB, Stupar RM, Specht JE, Graef GL, Lorenz AJ (2017) Dissecting the genetic basis of local adaptation in soybean. Sci Rep. https://doi.org/10.1038/ s41598-017-17342-w

Bayer PE, Valliyodan B, Hu H, Marsh JI, Yuan Y, Vuong TD, Patil G, Song Q, Batley J, Varshney RK, Lam HM, Edwards D, Nguyen HT (2021) Sequencing the USDA core soybean collection reveals gene loss during domestication and breeding. Plant Genome. https://doi.org/10.1002/tpg2.20109

Bolger AM, Lohse M, Usadel B (2014) Trimmomatic: a flexible trimmer for Illumina sequence data. Bioinformatics 30:2114-2120. https://doi.org/10.1093/bioinformatics/btu170

Bolon YT, Joseph B, Cannon SB, Graham MA, Diers BW, Farmer AD, May GD, Muehlbauer GJ, Specht JE, Tu ZJ, Weeks N, Xu WW, Shoemaker RC, Vance CP (2010) Complementary genetic and genomic approaches help characterize the linkage group I seed protein QTL in soybean. BMC Plant Biol. https://doi.org/ 10.1186/1471-2229-10-41

Bradbury PJ, Zhang Z, Kroon DE, Casstevens TM, Ramdoss Y, Buckler ES (2007) TASSEL: software for association mapping of complex traits in diverse samples. Bioinformatics 23:2633-2635. https://doi.org/10.1093/bioinformatics/btm308

Browning SR, Browning BL (2007) Rapid and accurate haplotype phasing and missing-data inference for whole-genome association studies by use of localized haplotype clustering. Am J Hum Genet 81:1084-1097. https://doi.org/10.1086/521987

Brzostowski LF, Pruski TI, Specht JE, Diers BW (2017) Impact of seed protein alleles from three soybean sources on seed composition and agronomic traits. Theor Appl Genet 130:2315-2326. https:// doi.org/10.1007/s00122-017-2961-x
Buels R, Yao E, Diesh CM, Hayes RD, Munoz-Torres M, Helt G, Goodstein DM, Elsik CG, Lewis SE, Stein L, Holmes IH (2016) JBrowse: a dynamic web platform for genome visualization and analysis. Genome Biol. https://doi.org/10.1186/ s13059-016-0924-1

Cao Y, Li S, Wang Z, Chang F, Kong J, Gai J, Zhao T (2017) Identification of major quantitative trait loci for seed oil content in soybeans by combining linkage and genome-wide association mapping. Front Plant Sci 8:1222. https://doi.org/10.3389/fpls.2017.01222

Chang HX, Lipka AE, Domier LL, Hartman GL (2016) Characterization of disease resistance loci in the USDA soybean germplasm collection using genome-wide association studies. Phytopathology 106:1139-1151. https://doi.org/10.1094/PHYTO-01-16-0042-FI

Cingolani P, Platts A, le Wang L, Coon M, Nguyen T, Wang L, Land SJ, Lu X, Ruden DM (2012) A program for annotating and predicting the effects of single nucleotide polymorphisms, SnpEff: SNPs in the genome of Drosophila melanogaster strain w1118; iso-2; iso-3. Fly (austin) 6:80-92. https://doi.org/10.4161/fly. 19695

Danecek P, Auton A, Abecasis G, Albers CA, Banks E, DePristo MA, Handsaker RE, Lunter G, Marth GT, Sherry ST, McVean G, Durbin R (2011) The variant call format and VCFtools. Bioinformatics 27:2156-2158. https://doi.org/10.1093/bioinformatics/btr330

Diers BW, Keim P, Fehr WR, Shoemaker RC (1992) RFLP analysis of soybean seed protein and oil content. Theor Appl Genet 83:608-612. https://doi.org/10.1007/bf00226905

Do TD, Vuong TD, Dunn D, Clubb M, Valliyodan B, Patil G, Chen P, Xu D, Nguyen HT, Shannon JG (2019) Identification of new loci for salt tolerance in soybean by high-resolution genome-wide association mapping. BMC Genom 20:318. https://doi.org/10. 1186/s12864-019-5662-9

Dong SS, He WM, Ji JJ, Zhang C, Guo Y, Yang TL (2020) LDBlockShow: a fast and convenient tool for visualizing linkage disequilibrium and haplotype blocks based on variant call format files. Brief Bioinform. https://doi.org/10.1093/bib/bbaa227

Endelman JB, Jannink JL (2012) Shrinkage estimation of the realized relationship matrix. G3 2:1405-1413. DOI: https://doi.org/10. 1534/g3.112.004259

Fang C, Ma Y, Wu S, Liu Z, Wang Z, Yang R, Hu G, Zhou Z, Yu H, Zhang M, Pan Y, Zhou G, Ren H, Du W, Yan H, Wang Y, Han D, Shen Y, Liu S, Liu T, Zhang J, Qin H, Yuan J, Yuan X, Kong F, Liu B, Li J, Zhang Z, Wang G, Zhu B, Tian Z (2017) Genomewide association studies dissect the genetic networks underlying agronomical traits in soybean. Genome Biol. https://doi.org/10. 1186/s13059-017-1289-9

Figura G, Koscianska E, Krzyzosiak WJ (2015) In vitro expansion of CAG, CAA, and mixed CAG/CAA repeats. Int J Mol Sci 16:18741-18751. https://doi.org/10.3390/ijms160818741

Fliege C (2019) Genomic changes underlying disease resistance and high protein QTL. University of Illinois at Urbana-Champaign, Illinois, p 136

Gabriel SB, Schaffner SF, Nguyen H, Moore JM, Roy J, Blumenstiel B, Higgins J, DeFelice M, Lochner A, Faggart M, Liu-Cordero SN, Rotimi C, Adeyemo A, Cooper R, Ward R, Lander ES, Daly MJ, Altshuler D (2002) The structure of haplotype blocks in the human genome. Science 296:2225-2229. https://doi.org/10.1126/ science. 1069424

Hwang EY, Song Q, Jia G, Specht JE, Hyten DL, Costa J, Cregan PB (2014) A genome-wide association study of seed protein and oil content in soybean. BMC Genom 15:1. https://doi.org/10.1186/ 1471-2164-15-1

Hyten DL, Song Q, Zhu Y, Choi IY, Nelson RL, Costa JM, Specht JE, Shoemaker RC, Cregan PB (2006) Impacts of genetic bottlenecks on soybean genome diversity. Proc Natl Acad Sci 103:1666616671. https://doi.org/10.1073/pnas.0604379103 
Jarquin D, Specht J, Lorenz A (2016) prospects of genomic prediction in the usda soybean germplasm collection: historical data creates robust models for enhancing selection of accessions. G3 Genes, Genom, Genet 6:2329-2341. https://doi.org/10.1534/g3. 116.031443

Kim MS, Lozano R, Kim JH, Bae DN, Kim ST, Park JH, Choi MS, Kim J, Ok HC, Park SK, Gore MA, Moon JK, Jeong SC (2021) The patterns of deleterious mutations during the domestication of soybean. Nat Commun. https://doi.org/10.1038/s41467-020-20337-3

Kofsky J, Zhang H, Song BH (2018) The untapped genetic reservoir: the past, current, and future applications of the wild soybean (Glycine soja). Front Plant Sci 9:949. https://doi.org/10.3389/ fpls.2018.00949

Korunes KL, Samuk K (2021) pixy: unbiased estimation of nucleotide diversity and divergence in the presence of missing data. Mol Ecol Resour 21:1359-1368. https://doi.org/10.1111/1755-0998.13326

Lee S, Van K, Sung M, Nelson R, LaMantia J, McHale LK, Mian MAR (2019) Genome-wide association study of seed protein, oil and amino acid contents in soybean from maturity groups I to IV. Theor Appl Genet 132:1639-1659. https://doi.org/10. 1007/s00122-019-03304-5

Li H (2013) Aligning sequence reads, clone sequences and assembly contigs with BWA-MEM. arXiv.

Li H, Handsaker B, Wysoker A, Fennell T, Ruan J, Homer N, Marth G, Abecasis G, Durbin R (2009) The Sequence Alignment/Map format and SAMtools. Bioinformatics 25:2078-2079. https:// doi.org/10.1093/bioinformatics/btp352

Liu X, Huang M, Fan B, Buckler ES, Zhang Z (2016) Iterative usage of fixed and random effect models for powerful and efficient genome-wide association studies. PLoS Genet. https://doi.org/ 10.1371/journal.pgen.1005767

Liu Y, Du H, Li P, Shen Y, Peng H, Liu S, Zhou G-A, Zhang H, Liu Z, Shi M, Huang X, Li Y, Zhang M, Wang Z, Zhu B, Han B, Liang C, Tian Z (2020) Pan-genome of wild and cultivated soybeans. Cell 182:162-176. https://doi.org/10.1016/j.cell. 2020.05 .023

Marsh JI, Hu H, Gill M, Batley J, Edwards D (2021) Crop breeding for a changing climate: integrating phenomics and genomics with bioinformatics. Theor Appl Genet 134:1677-1690. https://doi.org/ 10.1007/s00122-021-03820-3

McInnes L, Healy J, Saul N, Großberger L (2018) UMAP: uniform manifold approximation and projection. J Open Source Softw. https://doi.org/10.21105/joss.00861

McKenna A, Hanna M, Banks E, Sivachenko A, Cibulskis K, Kernytsky A, Garimella K, Altshuler D, Gabriel S, Daly M, DePristo MA (2010) The genome analysis toolkit: a mapreduce framework for analyzing next-generation DNA sequencing data. Genome Res 20:1297-1303. https://doi.org/10.1101/gr.107524.110

Nageshwaran S, Festenstein R (2015) Epigenetics and triplet-repeat neurological diseases. Front Neurol 6:262. https://doi.org/10. 3389/fneur.2015.00262

Nichols DM, Glover KD, Carlson SR, Specht JE, Diers BW (2006) Fine mapping of a seed protein QTL on soybean linkage group I and its correlated effects on agronomic traits. Crop Sci 46:834-839. https://doi.org/10.2135/cropsci2005.05-0168

Patil G, Vuong TD, Kale S, Valliyodan B, Deshmukh R, Zhu C, Wu X, Bai Y, Yungbluth D, Lu F, Kumpatla S, Shannon JG, Varshney RK, Nguyen HT (2018) Dissecting genomic hotspots underlying seed protein, oil, and sucrose content in an interspecific mapping population of soybean using high-density linkage mapping. Plant Biotechnol J 16:1939-1953. https://doi.org/10.1111/pbi.12929

Patil GB, Lakhssassi N, Wan J, Song L, Zhou Z, Klepadlo M, Vuong TD, Stec AO, Kahil SS, Colantonio V, Valliyodan B, Rice JH, Piya S, Hewezi T, Stupar RM, Meksem K, Nguyen HT (2019) Whole-genome re-sequencing reveals the impact of the interaction of copy number variants of the rhg 1 and Rhg 4 genes on broad-based resistance to soybean cyst nematode. Plant Biotechnol J 17:1595-1611. https://doi.org/10.1111/pbi.13086

Pawlowski ML, Vuong TD, Valliyodan B, Nguyen HT, Hartman GL (2020) Whole-genome resequencing identifies quantitative trait loci associated with mycorrhizal colonization of soybean. Theor Appl Genet 133:409-417. https://doi.org/10.1007/ s00122-019-03471-5

Pedersen BS, Quinlan AR (2018) Mosdepth: quick coverage calculation for genomes and exomes. Bioinformatics 34:867-868. https:// doi.org/10.1093/bioinformatics/btx699

Popovic V, Vidic M, Jockovic D, Ikanovic J, Jaksic S, Cvijanovic G (2012) Variability and correlations between yield components of soybean [Glycine max (L.) Merr.]. Genetika 44:33-45. https://doi. org/10.2298/gensr1201033p

Price AL, Patterson NJ, Plenge RM, Weinblatt ME, Shadick NA, Reich D (2006) Principal components analysis corrects for stratification in genome-wide association studies. Nat Genet 38:904-909. https://doi.org/10.1038/ng1847

Purcell S, Neale B, Todd-Brown K, Thomas L, Ferreira MAR, Bender D, Maller J, Sklar P, de Bakker PIW, Daly MJ, Sham PC (2007) PLINK: a tool set for whole-genome association and populationbased linkage analyses. Am J Human Genet 81:559-575. https:// doi.org/10.1086/519795

R Core Team (2021) R: A language and environment for statistical computing. R Foundation for Statistical Computing, Vienna, Austria.

Raj A, Stephens M, Pritchard JK (2014) fastSTRUCTURE: variational inference of population structure in large SNP data sets. Genetics 197:573-589. https://doi.org/10.1534/genetics.114.164350

Ray DK, Mueller ND, West PC, Foley JA (2013) Yield trends are insufficient to double global crop production by 2050. PLoS One. https://doi.org/10.1371/journal.pone.0066428

Reynolds M, Chapman S, Crespo-Herrera L, Molero G, Mondal S, Pequeno DNL, Pinto F, Pinera-Chavez FJ, Poland J, RiveraAmado C, Saint Pierre C, Sukumaran S (2020) Breeder friendly phenotyping. Plant Sci. https://doi.org/10.1016/j.plantsci.2019. 110396

Shacham T, Sharma N, Lederkremer GZ (2019) Protein misfolding and ER stress in Huntington's disease. Front Mol Biosci. https://doi. org/10.3389/fmolb.2019.00020

Tabib A, Vishwanathan S, Seleznev A, McKeown PC, Downing T, Dent C, Sanchez-Bermejo E, Colling L, Spillane C, Balasubramanian S (2016) A polynucleotide repeat expansion causing temperature-sensitivity persists in wild irish accessions of Arabidopsis thaliana. Front Plant Sci. https://doi.org/10.3389/fpls.2016.01311

Tardivel A, Torkamaneh D, Lemay M-A, Belzile F, O'Donoughue LS (2019) A systematic gene-centric approach to define haplotypes and identify alleles on the basis of dense single nucleotide polymorphism datasets. The Plant Genome. https://doi.org/10.3835/ plantgenome2018.08.0061

Tilman D, Balzer C, Hill J, Befort BL (2011) Global food demand and the sustainable intensification of agriculture. Proc Natl Acad Sci 108:20260-20264. https://doi.org/10.1073/pnas.1116437108

Torkamaneh D, Laroche J, Valliyodan B, O’Donoughue L, Cober E, Rajcan I, Vilela Abdelnoor R, Sreedasyam A, Schmutz J, Nguyen HT, Belzile F (2021a) Soybean (Glycine max) Haplotype Map (GmHapMap): a universal resource for soybean translational and functional genomics. Plant Biotechnol J 19:324-334. https://doi. org/10.1111/pbi.13466

Torkamaneh D, Lemay MA, Belzile F (2021b) The pan-genome of the cultivated soybean (PanSoy) reveals an extraordinarily conserved gene content. Plant Biotechnol J 19:1852-1862. https://doi.org/ 10.1111/pbi. 13600

Valliyodan B, Brown AV, Wang J, Patil G, Liu Y, Otyama PI, Nelson RT, Vuong T, Song Q, Musket TA, Wagner R, Marri P, Reddy S, Sessions A, Wu X, Grant D, Bayer PE, Roorkiwal M, Varshney 
RK, Liu X, Edwards D, Xu D, Joshi T, Cannon SB, Nguyen HT (2021) Genetic variation among 481 diverse soybean accessions, inferred from genomic re-sequencing. Sci Data 8:50. https://doi. org/10.1038/s41597-021-00834-w

Valliyodan B, Cannon SB, Bayer PE, Shu S, Brown AV, Ren L, Jenkins J, Chung CYL, Chan TF, Daum CG, Plott C, Hastie A, Baruch K, Barry KW, Huang W, Patil G, Varshney RK, Hu H, Batley J, Yuan Y, Song Q, Stupar RM, Goodstein DM, Stacey G, Lam HM, Jackson SA, Schmutz J, Grimwood J, Edwards D, Nguyen HT (2019) Construction and comparison of three reference-quality genome assemblies for soybean. Plant J 100:1066-1082. https:// doi.org/10.1111/tpj.14500

van Dijk M, Morley T, Rau ML, Saghai Y (2021) A meta-analysis of projected global food demand and population at risk of hunger for the period 2010-2050. Nat Food 2:494-501. https://doi.org/ 10.1038/s43016-021-00322-9

Vaughn JN, Nelson RL, Song Q, Cregan PB, Li Z (2014) The genetic architecture of seed composition in soybean is refined by genomewide association scans across multiple populations. G3 Genes, Genom, Genet 4:2283-2294. https://doi.org/10.1534/g3.114. 013433

Welch BL (1947) The generalisation of student's problems when several different population variances are involved. Biometrika 34:28-35. https://doi.org/10.1093/biomet/34.1-2.28

Yin L, Zhang H, Tang Z, Xu J, Yin D, Zhang Z, Yuan X, Zhu M, Zhao S, Li X, Liu X (2021) rMVP: a memory-efficient, visualizationenhanced, and parallel-accelerated tool for genome-wide association study. Genom Proteom Bioinform. https://doi.org/10.1016/j. gpb.2020.10.007
Zappia L, Oshlack A (2018) Clustering trees: a visualization for evaluating clusterings at multiple resolutions. Gigascience. https://doi. org/10.1093/gigascience/giy083

Zhang H, Mittal N, Leamy LJ, Barazani O, Song BH (2017) Back into the wild-apply untapped genetic diversity of wild relatives for crop improvement. Evol Appl 10:5-24. https://doi.org/10.1111/ eva.12434

Zhao S, Zheng F, He W, Wu H, Pan S, Lam HM (2015) Impacts of nucleotide fixation during soybean domestication and improvement. BMC Plant Biol. https://doi.org/10.1186/ s12870-015-0463-Z

Zhou Z, Jiang Y, Wang Z, Gou Z, Lyu J, Li W, Yu Y, Shu L, Zhao Y, Ma Y, Fang C, Shen Y, Liu T, Li C, Li Q, Wu M, Wang M, Wu Y, Dong Y, Wan W, Wang X, Ding Z, Gao Y, Xiang H, Zhu B, Lee SH, Wang W, Tian Z (2015) Resequencing 302 wild and cultivated accessions identifies genes related to domestication and improvement in soybean. Nat Biotechnol 33:408-414. https://doi. org/10.1038/nbt.3096

Zhu L, Wu H, Li H, Tang H, Zhang L, Xu H, Jiao F, Wang N, Yang L (2021) Short tandem repeats in plants: genomic distribution and function prediction. Electron J Biotechnol 50:37-44. https://doi. org/10.1016/j.ejbt.2020.12.003

Publisher's Note Springer Nature remains neutral with regard to jurisdictional claims in published maps and institutional affiliations. 